\title{
ANALYSIS OF THE JUDGMENT OF RELATIVE POSITION (Preliminary Communication)
}

BY

\author{
Wing Commander P. C. Livingston
}

MEDICAL BRANCH, R.A.F.

OwING to the interposition of mind in the reactive processes of the special senses, it is impossible to create values that are interchangeable between experimenters. The shape of objects, and the setting of them in the world about, is influenced so greatly by the nature of individual experience, that only the simplest mathematical configurations are capable of comparative representation through a common psycho-visual pathway. The effect of landscape upon a group of observers is sufficient testimony to the correctness of this statement. To one, the most impressive feature will be the hill in the distance, to another the spinney, to a third the pond. Where different objects in a scene become heavily accentuated, they tend to form points of reference to which the remaining objects radiate. Thus, without further consideration there is revealed a factor that seems to account for certain differences in the apportioning of space. Further, the tide of selectivity for detail may flow with considerable variation as to strength and induce as it were, +- phases. The observer who has spent his childhood in hilly surroundings acquires conversely a distaste for the desert. This is not because of an inherent negation for flatness, but because of an acutely sensitive memory of hills, whereby antagonism is induced. Sometimes, so strong are these + - phases that they can be projected into consciousness, and become the precursors of a neurosis. It is important to emphasise the points, because their influence may be carried into calculations that involve spatial judgment.

The object of this paper is to bring into the foreground certain features of binocular vision which do not appear to have received full recognition, and which, studied more fully, appear to have a close association with depth perception. Considered here, the term " depth perception" means the highest stratum of spatial recognition. While acknowledging that visual acuity and ocular muscle balance must play their part in shaping the framework to support the picture, they can exhibit deficiencies, which do not, however, detract from the full recognition of that quality recognisable as depth. It is considered that fine gradations in intrinsic or extrinsic muscle tone expressed as a kinetic stimulus assisting in the acute analysis of relative position do not possess 
the full power to express themselves with consistent accuracy. This preliminary investigation has been carried out with the aid of two pieces of apparatus specially designed to analyse what are felt to be the sensations directly involved.

\section{The Rotating Depth Perception Apparatus}

This instrument was devised with the object of examining the nature of depth perception in its pure form by the elimination of as many secondary considerations as possible. The test is based upon the three pins of Helmholtz, but it has been made so that the pins can be rotated in parallel through the full range of the perimeter. The pins themselves are blacked, and of $1 \mathrm{~mm}$. thickness. The background is a flat gray, and owing to the method of illumination, is entirely without shadows. There is no chance of using external points of reference. The observer's head is fixed in a head-rest and clamped to prevent artificial parallax, through conscious or subconscious lateral movements, and the cord by which the centre pin is moved is uniform in texture, and yields no clue. The distance between the two fixed pins and the observer is 11 feet. The range of movement of the centre pin is $240 \mathrm{~mm}$. Attached to the centre pin upon the adjustment of which the judgments are made is a stylo pen, which runs

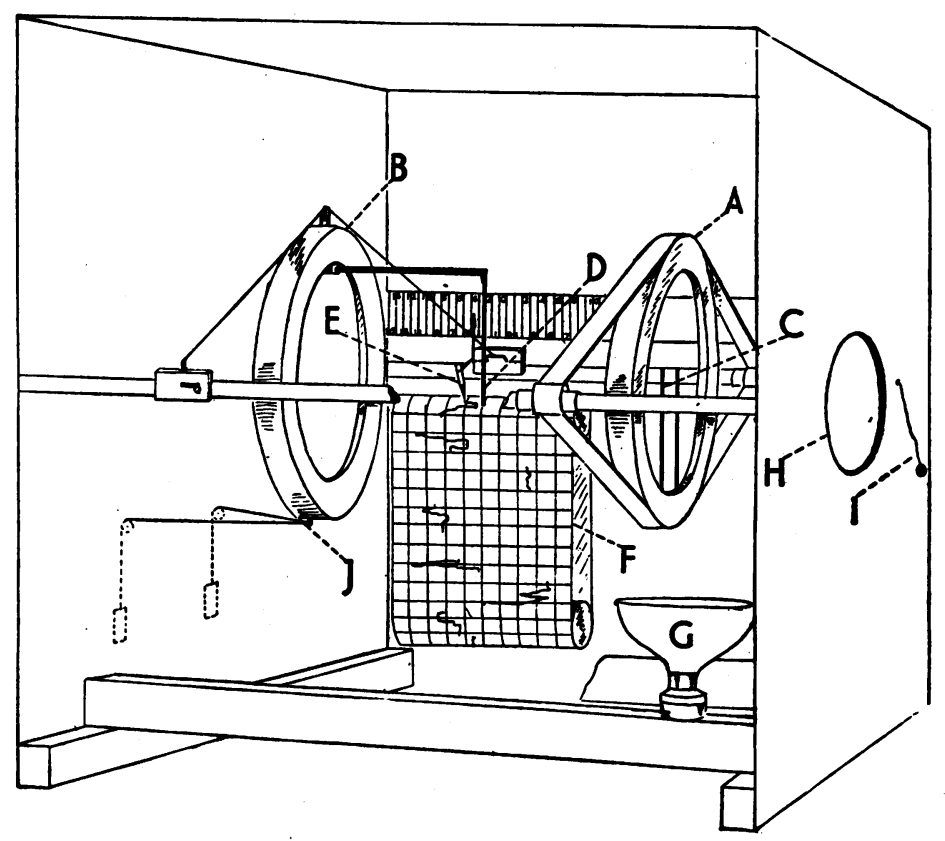


over graphed paper on a moving drum at a standard speed, so that all movements carried out in the process of re-setting the centre pin are recorded, and not merely the final judgment. This is a very important consideration. There is a special routine in adjusting the centre pin by the examiner, before each test. The points selected are, $+4-8+5-10$ measured in cms., and zero. The four selected positions or rotations are vertical, horizontal, and $15^{\circ}$ either side of horizontal. The examiner having set the centre pin according to this plan, asks the observer to move it back by means of his cord to what he feels is that position in which all three pins are equidistant from him. As four perimeter positions are employed, there are thus twenty separate tests to one graphic record. It is believed by this procedure that two visual characters are assessed, namely :

(i) The sensitivity of the retinae to stimulation at disparate points ;

(ii) The psycho-visual response to such stimuli which calls for interpretation varying in acuteness according to the rotated position of the pins.

In the vertical position the full interocular base line allows assessment under normal conditions, while in the horizontal a situation is presented that is impossible to analyse accurately. Graphic records show great differences in character between individuals in their endeavour to align the pins set horizontally. The rôle and importance of binocular vision is well demonstrated by the results obtained after moving the pins from the horizontal position to an angle of $15^{\circ}$ either side of horizontal, because there is often an immediate return of the power to analyse depth in a degree sometimes actually comparable to that which attends the setting of the pins in the normal, vertical, position. This is remarkable because, by a rotation from $180^{\circ} \rightarrow$ to $165^{\circ}$ or $15^{\circ}$, only a very small binocular factor has actually been regained. Further experiment tends to show, however, that cases arise in which, although disparate point retinal stimulation occurs, and binocular vision is present without restriction, yet it does not necessarily follow that depths can be accurately assessed. There is a third factor necessary to complete the whole, that is clearly demonstrated by the instrument to be described.

\section{The Rotating Stereogram and Pictures}

This instrument was designed for the purpose of studying the behaviour of the eyes to the presentation of dissimilar objects, their range of stereoscopic perception, and also for treating defects in ocular muscle balance. If the upper segment of the first picture 
of the series is examined through the Holmes stereoscope, it will be found arranged in the following fashion. The right eye sees a circle and dot, three horizontal parallel straight lines, and two triangles base to base. The left eye adds to this, two lines to the circle, three vertical parallel straight lines across the horizontal ones, and a dot in each triangle. Experiments with these charts have revealed clearly that the majority of observers experience alternate vision, and that there are marked variations in the quality alternation. This may be equally balanced, irregular, slow or rapid. The influence of the master eye may be very pronounced. It has been found that strong mastery tends to negative the faculty of depth perception, as also does the presence of a perfectly balanced

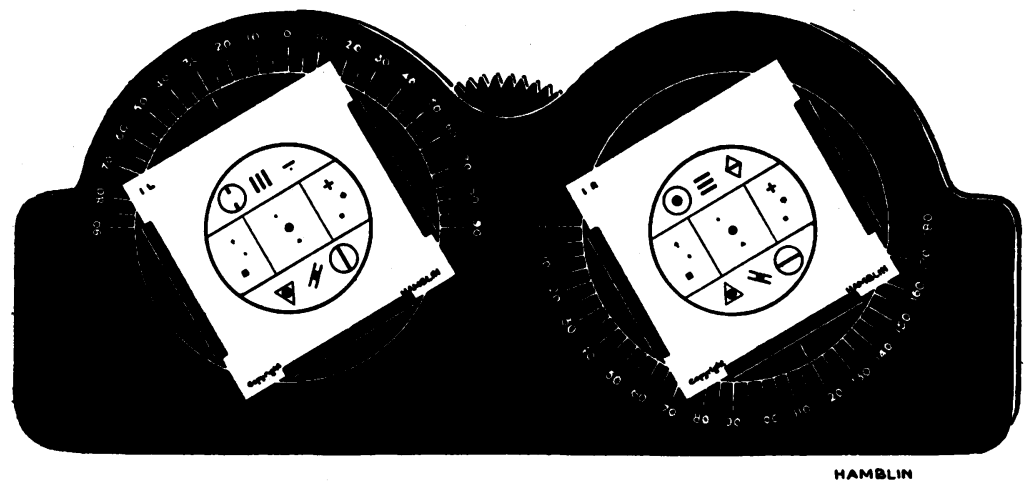

macular reception. These results seem to indicate that the comprehension of depth rests upon the presence of alternation, and upon the character and rhythm of that alternation. If this is so, then the processes concerned are dependent, not upon any specially attuned set of visual nerve fibres, nor upon an allotted cortical centre, but upon what might be termed sub-liminal alternate flicker. It is known that where one eye only is present accuracy in the spacing of objects depends largely upon lateral movements of the head. If the head is fixed in a vice, accuracy is at once considerably reduced. This habit of head shifting in those who have become uniocular is acquired naturally. So it seems that where a series of objects in space is regarded either predominantly through one eye or with perfect evenness of reception through both, the essential parallactic effect brought about by alternation is missing, and accurate analysis with regard to relative position becomes faulty.

It is possible by means of the rotating stereogram to develop the stereoscopic sense in those who at first have no acute understanding concerning relative position in space. The procedure is 
to work at first upon dissimilar objects and adjust the macular reception rate until a point is reached where marked neglect or long period alternation is replaced by rapid and equal periodicity. Attention is then given to the stereoscopic sections of the pictures. It will be found that having attacked unbalanced alternation, a situation is arrived at in which depth may now be appreciated. This achievement adds weight to the contention that alternation is essential for complete and accurate spatial orientation. Much work remains to be done with regard to the more detailed analysis of alternation in the optimum balance required to produce the greatest depth effect. It must be known what influences the master eye brings into the picture as a stabilising factor and centre of reference; and, what effects has the angle of separation of the line of vision of such an eye from the sagittal line.

To this end it is intended to carry these investigations through a large series of cases and in each instance to examine :-visual acuity with refraction; muscle balance including master eye and its relative ascendency over the other eye; depth perception as recorded by the rotating depth perception apparatus; and, the state of binocuiar reception and stereoscopic appreciation as studied with rotating stereogram.

\title{
ON DISABLEMENT AND SOCIAL CONDITIONS OF PATIENTS WITH PAST SYPHILITIC INTERSTITIAL KERATITIS*
}

BY

\author{
Esther DalsgaARD-Nielsen, M.D.
}

COPENHAGEN

I HAVE examined material comprising 173 patients with past interstitial keratitis. Of these patients 124 answered a call for re-examination while 49 were encountered accidentally in the course of this work. As to the gathering of this material I beg to refer to my book: "Keratitis parenchymatosa luica og dens Fœlgetilstande."1

The average age of these 173 patients was 32 years, with an average observation period of 20 years, the longest observation period covering 56 years. In the present paper I shall try to give an account of my findings concerning the disablement and social conditions of these patients.

\footnotetext{
* From the Departments of Ophthalmology of the Rigshospital (University Clinic) and the Kommunehospital (City Hospital), Copenhagen.
} 